\title{
Performance Analysis for a Fully Decentralized Transmit Power Allocation Scheme for Relay-Assisted Cognitive-Radio Systems ${ }^{\dagger}$
}

\author{
Jan Mietzner, Lutz Lampe, and Robert Schober \\ Dept. of Elec. \& Comp. Engineering, University of British Columbia, e-mail: \{janm,lampe,rschober $\}$ ece.ubc.ca
}

\begin{abstract}
We analyze the performance of a fully decentralized (FD) transmit power allocation (TPA) scheme for relay-assisted cognitive-radio (CR) systems. In particular, we assume that the frequency band chosen by the CR relay network for unlicensed spectrum usage overlaps with an active primary narrowband link. The considered FD-TPA scheme maximizes the signal-to-noiseplus-interference ratio at the destination node of the CR network according to a best-effort strategy, while limiting the interference experienced by the primary receiver. Numerical performance results show substantial improvements compared to non-cooperative transmission. Moreover, the performance of the FD-TPA scheme is close to that of the optimal centralized power allocation solution.
\end{abstract}

\section{INTRODUCTION}

C OGNITIVE radio (CR) systems have recently attracted considerable interest in the wireless community [1]. Traditionally, radio spectrum usage has been organized according to fixed frequency plans defined through government licenses. As opposed to this, CR systems are envisioned to take advantage of unused or partially occupied bands in an adaptive, dynamic, and unlicensed ('secondary') fashion, so as to allow for a more efficient spectrum utilization. To this end, CR systems will require spectrum-sensing [2] and radio-scene-analysis capabilities [1], based on which they will adjust key transmission parameters such as frequency bands and radiated transmit power. For example, CR capabilities will become relevant for ultra-wideband (UWB) radio systems [3]. In this paper, we focus on CR networks consisting of a possibly large number of low-power transceivers for short-range transmission (on the order of a couple of meters). Such a setup is, for example, relevant for wireless sensor networks (WSNs) and future personal area networks (PANs).

In order to achieve connectivity and guarantee a certain quality of service for such networks, (cooperative) relaying techniques appear to be an attractive choice. Available relays can either be dedicated cognitive relays or temporarily inactive cognitive devices acting as relays to assist the (current) sourcedestination link. So far, only a few papers on relaying techniques have been published that explicitly incorporate CR concepts. In [4], [5] focus was on the case where the individual relays operate in unused frequency bands only ('spectrum holes'). In our previous work [6], we proposed a fully decentralized (FD) transmit power allocation (TPA) scheme for relay-assisted $\mathrm{CR}$ systems, for the case where the frequency band chosen for unlicensed usage is not completely unoccupied, but accommodates an active primary narrowband link. For a similar scenario, a centralized algorithm for optimum relay selection was recently proposed in [7].

The FD-TPA scheme [6] maximizes the performance of the $\mathrm{CR}$ system, while guaranteeing that a certain pre-defined maximum interference level experienced by the primary receiver is

\footnotetext{
${ }^{\dagger}$ This work was supported by a postdoctoral fellowship from the German Academic Exchange Service (DAAD).
}

not exceeded. It requires a minimum of interaction between the CR nodes and can be performed solely by the relays, i.e., without any feedback information from the destination node. In [6], focus was on a pure maximization of the output signal-to-noiseplus-interference ratio (SINR) at the destination node of the CR network. However, for practical applications it is typically sufficient to achieve a certain target SINR value at the destination node, thus reducing the energy consumption at the relays. Second, for simplicity the impact of the primary transmitter on the performance of the CR system was neglected in [6]. Finally, the performance of the proposed FD-TPA scheme was assessed solely based on simulation results. In this paper, we

(i) generalize the FD-TPA scheme such that relaying is performed only if a pre-defined target SINR value is not accomplished by the source-destination link alone,

(ii) include the impact of the primary transmitter on the performance of the CR system,

(iii) provide a thorough performance analysis, which reveals that substantial performance improvements in comparison to non-cooperative transmission are achieved by the (generalized) FD-TPA scheme.

As will be seen, the performance analysis turns out to be rather involved, which is mainly due to the generalization of the FDTPA scheme in (i). In particular, our analysis requires the numerical evaluation of certain integral expressions, since closedform expressions do not seem feasible. Simulative performance results presented corroborate our analysis and show that the performance of the FD-TPA scheme is, in fact, close to that of the optimal centralized (OC) power allocation solution.

The paper is organized as follows: In Section II, the system model and the optimization problem under consideration are introduced and the (generalized) FD-TPA scheme is described. In Section III, the performance analysis for the FD-TPA scheme is presented, and numerical performance results are provided in Section IV. Conclusions are offered in Section V.

\section{System Model}

We consider a short-range relay-assisted wideband or UWB CR system that is based on code-division multiple access (CDMA). The source-destination node pair (S-D) is assisted by $N_{\mathrm{r}}$ perfectly synchronized (in time and frequency) relay nodes $\mathrm{R}_{i}$ $\left(i=1, \ldots, N_{\mathrm{r}}\right)$, which are equipped with mutually orthogonal spreading codes (spreading length $N_{\mathrm{sp}}$ ). For simplicity and practical relevance, we assume that all CR nodes employ a single antenna. Throughout this paper, we assume that a primary transmitter-receiver pair $\mathrm{U}_{\mathrm{tx}}-\mathrm{U}_{\mathrm{rx}}$, e.g., a wireless local area network (WLAN) system, is present in the vicinity of the CR system, operating within a frequency band that fully overlaps with the band chosen by the CR system. The bandwidth $B_{\mathrm{U}}$ occupied by the primary link is assumed to be small compared to the bandwidth $B_{\mathrm{CR}}$ of the CR system, whereas the average transmit power $P_{\mathrm{U}_{\mathrm{tx}}}$ employed by the primary transmitter is assumed to be much larger than the maximum transmit 
powers $P_{\mathrm{S}, \max }$ and $P_{\mathrm{R}_{i} \text {, max }}$ available at the CR nodes. Finally, the primary system is assumed to employ a time-division duplex (TDD) mode using identical frequency bands on the forward and the reverse link. ${ }^{1}$ In the sequel, the bandwidth ratio $B_{\mathrm{U}} / B_{\mathrm{CR}}$ is denoted by $\rho$, and the maximum sum interference power tolerated by the primary receiver is denoted by $\xi$.

The channel impulse response (CIR) associated with a certain link $\mathrm{X} \rightarrow \mathrm{Y}$ from one node $\mathrm{X}$ to another node $\mathrm{Y}$, where $\mathrm{X}, \mathrm{Y} \in\left\{\mathrm{S}, \mathrm{D}, \mathrm{R}_{1}, \ldots, \mathrm{R}_{N_{\mathrm{r}}}, \mathrm{U}_{\mathrm{tx}}, \mathrm{U}_{\mathrm{rx}}\right\}$, is in the following denoted as $\mathbf{h}_{\mathrm{X}, \mathrm{Y}}:=\left[h_{\mathrm{X}, \mathrm{Y}}^{(0)}, \ldots, h_{\mathrm{X}, \mathrm{Y}}^{\left(L_{\mathrm{X}, \mathrm{Y}}\right)}\right]^{\mathrm{T}}$, where $L_{\mathrm{X}, \mathrm{Y}}$ denotes the corresponding channel memory length. Moreover, we define the channel energy $\alpha_{\mathrm{X}, \mathrm{Y}}:=\sum_{l=0}^{L_{\mathrm{X}, \mathrm{Y}}}\left|h_{\mathrm{X}, \mathrm{Y}}^{(l)}\right|^{2}$. Since the bandwidth $B_{\mathrm{U}}$ is assumed to be comparatively small, all links associated with the primary transmitter/ receiver are modeled with a channel memory length of zero. The system model under consideration is illustrated in Fig. 1.

Throughout this paper, a quasi-static scenario is considered. The destination node $\mathrm{D}$ is assumed to have perfect knowledge of the CIRs $\mathbf{h}_{\mathrm{S}, \mathrm{D}}$ and $\mathbf{h}_{\mathrm{R}_{i}, \mathrm{D}}$ associated with the sourcedestination and the relay-destination links $\left(i=1, \ldots, N_{\mathrm{r}}\right)$. Similarly, each relay node $\mathrm{R}_{i}$ is assumed to have perfect knowledge of the CIR $\mathbf{h}_{\mathrm{S}, \mathrm{R}_{i}}$ and the CIRs $\mathbf{h}_{\mathrm{R}_{i^{\prime}}, \mathrm{R}_{i}}$ associated with the links from the other relays $\mathrm{R}_{i^{\prime}}\left(i^{\prime} \neq i\right)$ to itself. Furthermore, it is assumed that based on built-in radio-scene-analysis functionalities the cognitive source node and the cognitive relays are aware of the channel energies $\alpha_{\mathrm{S}, \mathrm{U}_{\mathrm{rx}}}$ or $\alpha_{\mathrm{R}_{i}, \mathrm{U}_{\mathrm{rx}}}$ associated with its own link in direction of the primary receiver. This requires some acquisition phase, before the relay-assisted CR network can start to operate. In this context, it is assumed that the primary transmitter and receiver change their roles every now and then. Moreover, it is assumed that $\alpha_{\mathrm{S}, \mathrm{U}}=\alpha_{\mathrm{U}, \mathrm{S}}$ and $\alpha_{\mathrm{R}_{i}, \mathrm{U}}=\alpha_{\mathrm{U}, \mathrm{R}_{i}}\left(i=1, \ldots, N_{\mathrm{r}}\right)$, which is reasonable for primary systems operating in a TDD mode. ${ }^{2}$

\section{A. Transmission Protocol}

The transmission protocol under consideration consists of two orthogonal time slots. Within the first time slot, the source node $\mathrm{S}$ broadcasts a message to the relay nodes $\mathrm{R}_{1}, \ldots, \mathrm{R}_{N_{\mathrm{r}}}$ and the destination node $\mathrm{D}$, while the transmit power $P_{\mathrm{S}}$ is adjusted such that the interference constraint is met, i.e., $\rho P_{\mathrm{S}} \alpha_{\mathrm{S}, \mathrm{U}_{\mathrm{rx}}} \leq \xi$. Furthermore, $P_{\mathrm{S}}$ is limited by the maximum available transmit power $P_{\mathrm{S}, \max }$ :

$$
P_{\mathrm{S}}:=\min \left\{P_{\mathrm{S}, \max }, \frac{\xi}{\rho \alpha_{\mathrm{S}, \mathrm{U}_{\mathrm{rx}}}}\right\} .
$$

Throughout this paper, we assume that the source node transmits a large number of short messages using a low duty cycle (as it is typical, e.g., for WSN applications), so that a relaying phase can be accommodated in the time domain without causing a critical rate loss. When receiving, the cognitive nodes are assumed to employ simple despreading for interference suppression (rather than more sophisticated filtering techniques). Correspondingly, the primary interference power appears at cognitive node $\mathrm{Y}$ as additive white Gaussian noise (AWGN) with

\footnotetext{
${ }^{1}$ TDD is becoming increasingly popular and has been adopted as the only or one possible option, e.g., in IEEE 802.11 WLAN systems [8, Ch. 24].

${ }^{2}$ In order to estimate the channel energies from the signal strengths received from a primary transmitter, the (average) transmit power $P_{\mathrm{U}_{\mathrm{tx}}}$ must be known. This appears to be a reasonable assumption, since due to the fixed frequency plans associated with primary spectrum usage it is known which systems will operate in the frequency band under consideration.
}

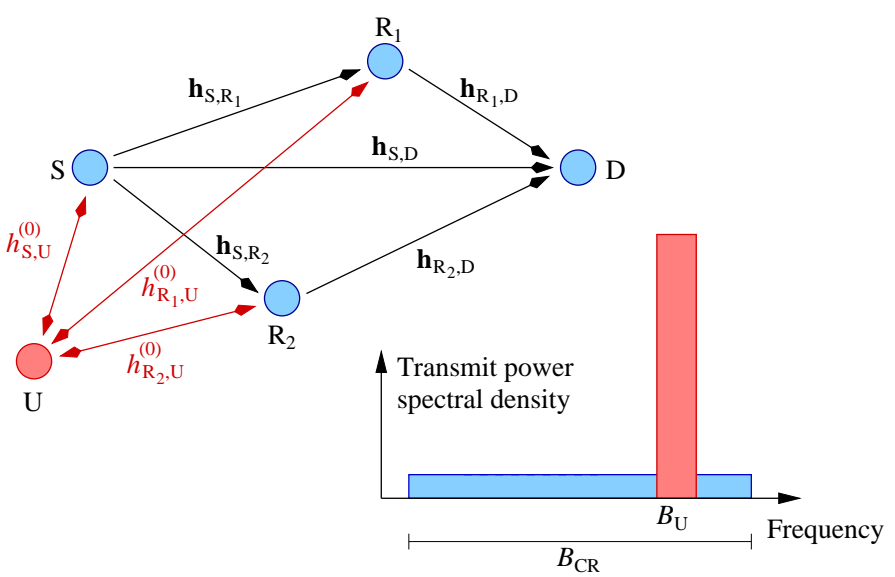

Fig. 1. System model under consideration and interference scenario in the frequency domain (for the example of two relays and a single primary transmitter or receiver).

variance $\sigma_{\mathrm{i}, \mathrm{Y}}^{2}:=\frac{1}{N_{\mathrm{sp}}} P_{\mathrm{U}_{\mathrm{tx}}} \alpha_{\mathrm{U}_{\mathrm{tx}}, \mathrm{Y}}[9, \mathrm{Ch} .13 .2]$. Moreover, the destination node and each relay node are assumed to employ a Rake receiver, which performs optimal maximum-ratio combining (MRC) of the signal received from the source node. In the sequel, let $\gamma_{D}$ denote the (overall) MRC output SINR at the destination node. Moreover, let $\sigma_{n, Y}^{2}$ denote the variance of the AWGN process at cognitive node Y. If the MRC output SINR $\gamma_{\mathrm{D}}$ exceeds a certain pre-defined target SINR value $\gamma_{\mathrm{D} \text {, target }}$ after completion of the source transmission phase, i.e.,

$$
\gamma_{\mathrm{D}}=\gamma_{\mathrm{S} \rightarrow \mathrm{D}}:=\frac{P_{\mathrm{S}} \alpha_{\mathrm{S}, \mathrm{D}}}{\sigma_{\mathrm{i}, \mathrm{D}}^{2}+\sigma_{\mathrm{n}, \mathrm{D}}^{2}} \geq \gamma_{\mathrm{D}, \text { target }},
$$

the destination node broadcasts a short acknowledgment (ACK) signal to inform the source node and the relay nodes that relaying is not required. ${ }^{3}$ Otherwise, the relaying process is initiated.

In the latter case, all relays that receive the message from the source node with an MRC output SINR of

$$
\gamma_{\mathrm{S} \rightarrow \mathrm{R}_{i}}:=\frac{P_{\mathrm{S}} \alpha_{\mathrm{S}, \mathrm{R}_{i}}}{\sigma_{\mathrm{i}, \mathrm{R}_{i}}^{2}+\sigma_{\mathrm{n}, \mathrm{R}_{i}}^{2}} \geq \gamma_{\mathrm{th}},
$$

where $\gamma_{\text {th }}$ denotes some threshold SINR value, are assumed to decode the message without any errors. These $N_{\mathrm{r}}^{\prime} \leq N_{\mathrm{r}}$ relays then broadcast a short ACK signal to inform the other relays and the destination node that they will participate in the upcoming relaying phase. ${ }^{4}$ In the sequel, let $\Pi_{\mathrm{r}} \subseteq\left\{1, \ldots, N_{\mathrm{r}}\right\}$ denote the index set associated with the participating relays, where $\left|I_{\mathrm{r}}\right|=N_{\mathrm{r}}^{\prime}$. Within the second time slot, the $N_{\mathrm{r}}^{\prime}$ relays re-encode the message (using the orthogonal spreading codes) and simultaneously retransmit it, and the destination node performs optimal MRC of the corresponding received signals, respectively. In this context, we define

$$
\gamma_{\mathrm{R}_{i} \rightarrow \mathrm{D}}:=\frac{P_{\mathrm{R}_{i}} \alpha_{\mathrm{R}_{i}, \mathrm{D}}}{\sigma_{\mathrm{i}, \mathrm{D}}^{2}+\sigma_{\mathrm{n}, \mathrm{D}}^{2}}, \quad \gamma_{\mathrm{R} \rightarrow \mathrm{D}}:=\sum_{i \in \mathbb{I}_{\mathrm{r}}} \gamma_{\mathrm{R}_{i} \rightarrow \mathrm{D}},
$$

where $P_{\mathrm{R}_{i}}$ is the transmit power of relay $\mathrm{R}_{i}$. The MRC output SINR at the destination is thus given by $\gamma_{\mathrm{D}}=\gamma_{\mathrm{S} \rightarrow \mathrm{D}}+\gamma_{\mathrm{R} \rightarrow \mathrm{D}}$.

\footnotetext{
${ }^{3}$ Throughout this paper, we assume that the energy consumption of ACK signals is negligible. Moreover, we assume that ACK signals are sufficiently protected using some low-rate channel code, so that they can be received reliably throughout the entire CR network.

${ }^{4}$ Since the relays are equipped with orthogonal spreading codes, one-bit ACK signals are sufficient in order to be able to identify the participating relays.
} 


\section{B. Optimization Problem}

Assuming that no additional feedback information from the destination is available, our objective is to adjust the transmit powers $P_{\mathrm{R}_{i}}$ of the participating relays such that the MRC output SINR $\gamma_{\mathrm{R} \rightarrow \mathrm{D}}$ within the relaying phase is maximized, subject to the constraints that

(a) the sum interference power experienced by the primary receiver $\mathrm{U}_{\mathrm{rx}}$ in the second time slot remains smaller than $\xi$,

(b) the transmit power of each individual relay node does not exceed the maximum transmit power $P_{\mathrm{R}_{i} \text {,max }}$.

Correspondingly, the optimal (centralized) transmit power allocation results from the following linear program, which can, for example, be solved using the well-known simplex algorithm [10, Ch. 4]:

$$
\begin{array}{ll}
\text { maximize } & \gamma_{\mathrm{R} \rightarrow \mathrm{D}}=\frac{1}{\sigma_{\mathrm{i}, \mathrm{D}}^{2}+\sigma_{\mathrm{n}, \mathrm{D}}^{2}} \sum_{i \in \mathbb{I}_{\mathrm{r}}} P_{\mathrm{R}_{i}} \alpha_{\mathrm{R}_{i}, \mathrm{D}} \\
\text { subject to } & \rho \sum_{i \in \mathbb{I}_{\mathrm{r}}} P_{\mathrm{R}_{i}} \alpha_{\mathrm{R}_{i}, \mathrm{U}_{\mathrm{rx}}} \leq \xi \\
& P_{\mathrm{R}_{i}} \leq P_{\mathrm{R}_{i}, \max } \quad \text { for all } i \in \mathbb{I}_{\mathrm{r}} .
\end{array}
$$

We assume that $\sigma_{\mathrm{i}, \mathrm{D}}^{2}$ and $\sigma_{\mathrm{n}, \mathrm{D}}^{2}\left(\right.$ or $\left.\sigma_{\mathrm{i}, \mathrm{D}}^{2}+\sigma_{\mathrm{n}, \mathrm{D}}^{2}\right)$ have been measured beforehand and are perfectly known at the destination node. Moreover, we assume that the maximum transmit power levels $P_{\mathrm{R}_{i} \text {, max }}\left(i=1, \ldots, N_{\mathrm{r}}\right)$, the maximum tolerated interference power $\xi$, as well as the parameter $\rho$ are known throughout the CR network. Further details about the above optimization problem can be found in [6].

\section{Fully Decentralized Transmit Power Allocation Scheme}

In order to solve the above optimization problem, a central network node $\mathrm{C}$ would be required (e.g., the destination node or one of the relays) which needs to be aware of all channel energies $\alpha_{\mathrm{R}_{i}, \mathrm{D}}$ and $\alpha_{\mathrm{R}_{i}, \mathrm{U}_{\mathrm{rx}}}\left(i \in I_{\mathrm{r}}\right)$. After computing the optimal solution, node $\mathrm{C}$ would then forward the resulting transmit power levels to the participating relay nodes. Obviously, this requires a significant amount of overhead, especially since each relay node $\mathrm{R}_{i}$ needs to communicate its own channel energies $\alpha_{\mathrm{R}_{i}, \mathrm{D}}$ and $\alpha_{\mathrm{R}_{i}, \mathrm{U}_{\mathrm{rx}}}$ to the central node C. In the following, we consider a simple fully decentralized (FD) transmit power allocation (TPA) scheme, which neither requires an exchange of channel information nor of transmit power levels.

Based on the ACK signals broadcasted by the relays (cf. Section II-A), the number $N_{\mathrm{r}}^{\prime}$ of participating relays is known throughout the CR network. Moreover, each relay node $\mathrm{R}_{i}$ is assumed to be aware of the channel energy $\alpha_{\mathrm{R}_{i}, \mathrm{U}_{\mathrm{rx}}}$ associated with its own link in direction of the primary receiver. Correspondingly, each relay can adjust its transmit power level as

$$
P_{\mathrm{R}_{i}}:=\min \left\{P_{\mathrm{R}_{i}, \max }, \frac{\xi}{\rho N_{\mathrm{r}}^{\prime} \alpha_{\mathrm{R}_{i}, \mathrm{U}_{\mathrm{rx}}}}\right\},
$$

similar to (1). This guarantees that the primary receiver experiences a sum interference power of at most $\xi$, without any further interaction between the relays. For example, in the special case where $\xi /\left(\rho N_{\mathrm{r}}^{\prime} \alpha_{\mathrm{R}_{i}, \mathrm{U}_{\mathrm{rx}}}\right) \leq P_{\mathrm{R}_{i}, \max }$ for all $i \in I_{\mathrm{r}}$, each relay will cause an interference power of exactly $\xi / N_{\mathrm{r}}^{\prime}$. Moreover, due to the minimization in (6) it is guaranteed that the maximum transmit power available at each relay is not exceeded.

Note that for large distances of the primary receiver (i.e., $\alpha_{\mathrm{R}_{i}, \mathrm{U}_{\mathrm{rx}}} \rightarrow 0$ for all indices $i \in \Pi_{\mathrm{r}}$ ), (6) approaches the optimum centralized solution, since all active relays are able to

\begin{tabular}{|c|c|}
\hline $\bar{\gamma}_{l, \mathrm{X} \rightarrow \mathrm{Y}}$ & $\bar{\gamma}_{l, \mathrm{X} \rightarrow \mathrm{Y}}=P_{\mathrm{X}} \sigma_{l, \mathrm{X}, \mathrm{Y}}^{2}\left(\sigma_{\mathrm{i}, \mathrm{Y}}^{2}+\sigma_{\mathrm{n}, \mathrm{Y}}^{2}\right)^{-1}$ \\
\hline$w_{l, \mathrm{X}, \mathrm{Y}}$ & $w_{l, \mathrm{X}, \mathrm{Y}}=\prod_{\substack{l^{\prime}=0 \\
l^{\prime} \neq l}}^{L_{\mathrm{X}, \mathrm{Y}}} \frac{\sigma_{l, \mathrm{X}, \mathrm{Y}}^{2}}{\sigma_{l, \mathrm{X}, \mathrm{Y}}^{2}-\sigma_{l^{\prime}, \mathrm{X}, \mathrm{Y}}^{2}}$ \\
\hline$\varphi_{\mathrm{X}, \mathrm{Y}}$ & $\varphi_{\mathrm{X}, \mathrm{Y}}=\sum_{l=0}^{L_{\mathrm{X}, \mathrm{Y}}} w_{l, \mathrm{X}, \mathrm{Y}}$ \\
\hline$C_{1, \mathrm{Y}}$ & $C_{1, \mathrm{Y}}=N_{\mathrm{sp}}\left(P_{\mathrm{U}_{\mathrm{tx}}} \sigma_{0, \mathrm{U}_{\mathrm{tx}}, \mathrm{Y}}^{2}\right)^{-1}$ \\
\hline$C_{2, l, \mathrm{R}_{i}}$ & $C_{2, l, \mathrm{R}_{i}}=\gamma_{\mathrm{th}}\left(P_{\mathrm{S}} \sigma_{l, \mathrm{~S}, \mathrm{R}_{i}}^{2}\right)^{-1}$ \\
\hline$C_{3, l, \lambda, \mathrm{R}_{i}}$ & $C_{3, l, \lambda, \mathrm{R}_{i}}=\bar{\gamma}_{\lambda, \mathrm{R}_{i} \rightarrow \mathrm{D}}^{-1}-\bar{\gamma}_{l, \mathrm{~S} \rightarrow \mathrm{D}}^{-1}$ \\
\hline$C_{4, \mathrm{X}}$ & $C_{4, \mathrm{X}}=\xi\left(\rho \sigma_{0, \mathrm{X}, \mathrm{U}_{\mathrm{rx}}}^{2}\right)^{-1}$ \\
\hline$K_{l, \mathrm{R}_{i}}$ & $K_{l, \mathrm{R}_{i}}=\mathrm{e}^{-C_{2, l, \mathrm{R}_{i}} \sigma_{\mathrm{n}, \mathrm{R}_{i}}^{2}\left(1+C_{2, l, \mathrm{R}_{i}} / C_{1, \mathrm{R}_{i}}\right)}$ \\
\hline$\tilde{w}_{l, \mathrm{R}_{i}, \mathrm{D}}$ & $\tilde{w}_{l, \mathrm{R}_{i}, \mathrm{D}}=\prod_{\substack{i^{\prime} \in I_{\mathrm{r}}^{(\kappa)} \\
\left(i^{\prime}, l^{\prime}\right) \neq(i, l)}} \prod_{l^{\prime}=0}^{L_{\mathrm{R}, \mathrm{D}}} \frac{\bar{\gamma}_{l, \mathrm{R}_{i} \rightarrow \mathrm{D}}}{\bar{\gamma}_{l, \mathrm{R}_{i} \rightarrow \mathrm{D}}-\bar{\gamma}_{l^{\prime}, \mathrm{R}_{i^{\prime}} \rightarrow \mathrm{D}}}$ \\
\hline$v_{l, \lambda, i}$ & $v_{l, \lambda, i}=\frac{1}{C_{3, l, \lambda, \mathrm{R}_{i}}} \frac{w_{l, \mathrm{~S}, \mathrm{D}}}{\bar{\gamma}_{l, \mathrm{~S} \rightarrow \mathrm{D}}} \frac{\tilde{w}_{\lambda, \mathrm{R}_{i}, \mathrm{D}}}{\bar{\gamma}_{\lambda, \mathrm{R}_{i} \rightarrow \mathrm{D}}}$ \\
\hline$K_{\alpha}$ & $K_{\alpha}=\left(\sum_{l=0}^{L_{\mathrm{S}, \mathrm{D}}} w_{l, \mathrm{~S}, \mathrm{D}} \exp \left(-\gamma_{\mathrm{D}, \text { target }} / \bar{\gamma}_{l, \mathrm{~S} \rightarrow \mathrm{D}}\right)\right)$ \\
\hline$K_{\beta}$ & $K_{\beta}=K_{\alpha}\left(K_{\alpha} \varphi_{\mathrm{S}, \mathrm{D}}-1\right)$ \\
\hline
\end{tabular}
operate at their maximum transmit power level $P_{\mathrm{R}_{i}, \text { max }}$.
TABLE I

OVERVIEW OF CONSTANTS AND SPECIAL NOTATIONS

\section{PERformance AnAlysis For the FD-TPA Scheme}

It is desirable to have analytical expressions that allow us to assess the performance of the FD-TPA scheme and highlight its advantage over non-cooperative transmission (i.e., without relay assistance). To this end, we derive an expression for the cumulative distribution function $(\mathrm{CDF})$ of the overall MRC output SINR $\gamma_{\mathrm{D}}$ at the destination node, while treating the channel energies $\alpha_{\mathrm{S}, \mathrm{D}}, \alpha_{\mathrm{S}, \mathrm{R}_{i}}, \alpha_{\mathrm{R}_{i}, \mathrm{D}}\left(i \in I_{\mathrm{r}}\right), \alpha_{\mathrm{S}, \mathrm{U}_{\mathrm{rx}}}, \alpha_{\mathrm{R}_{i}, \mathrm{U}_{\mathrm{rx}}}, \alpha_{\mathrm{U}_{\mathrm{tx}}, \mathrm{R}_{i}}$, and $\alpha_{\mathrm{U}_{\mathrm{tx}}, \mathrm{D}}$ as statistically independent random variables.

In the following, all transmission links are assumed to be subject to quasi-static Rayleigh fading, i.e., the channel coefficients $h_{\mathrm{X}, \mathrm{Y}}^{(l)}\left(l=0, \ldots, L_{\mathrm{X}, \mathrm{Y}}\right)$ are complex Gaussian random variables with zero mean and variance $\sigma_{l, \mathrm{X}, \mathrm{Y}}^{2}$. In order to account for different link lengths, we assume a path-loss exponent of $p$. Correspondingly, the channel variances $\sigma_{l, \mathrm{X}, \mathrm{Y}}^{2}$ are modeled as $\sigma_{l, \mathrm{X}, \mathrm{Y}}^{2}:=\tilde{\sigma}_{l, \mathrm{X}, \mathrm{Y}}^{2}\left(d_{\mathrm{ref}} / d_{\mathrm{X}, \mathrm{Y}}\right)^{p}$, where $\sum_{l=0}^{L_{\mathrm{X}, \mathrm{Y}}} \tilde{\sigma}_{l, \mathrm{X}, \mathrm{Y}}^{2}=: 1, d_{\mathrm{X}, \mathrm{Y}}$ denotes the distance between node $\mathrm{X}$ and node $\mathrm{Y}$, and $d_{\text {ref }}$ denotes some reference link length.

With regard to the relaying process, we distinguish the following two cases:

( $\alpha)$ Event $\mathcal{E}^{(\alpha)}$ : The source node is able to accomplish the desired target SINR $\gamma_{\mathrm{D} \text {,target }}$ on its own (cf. (2)), i.e., relaying is not required $\left(\gamma_{\mathrm{S} \rightarrow \mathrm{D}} \geq \gamma_{\mathrm{D} \text {, target }}\right)$.

( $\beta)$ Event $\mathcal{E}^{\left(\beta_{\kappa}\right)}$ : The source node is not able to accomplish the target SINR $\gamma_{\mathrm{D} \text {,target }}$ on its own $\left(\gamma_{\mathrm{S} \rightarrow \mathrm{D}}<\gamma_{\mathrm{D} \text {,target }}\right)$, and a relaying process with $N_{\mathrm{r}}^{\prime} \leq N_{\mathrm{r}}$ active relays is initiated. The number of all possible index sets $I_{\mathrm{r}} \subseteq\left\{1, \ldots, N_{\mathrm{r}}\right\}$ of active relays (including the empty set $\Pi_{\mathrm{r}}=\emptyset$ ) is given by $\psi:=\sum_{i=0}^{N_{\mathrm{r}}}\left(\begin{array}{c}N_{\mathrm{r}} \\ i\end{array}\right)$. The $\kappa$ th index set is denoted as $\Pi_{\mathrm{r}}^{(\kappa)}$ $(\kappa \in\{0, \ldots, \psi-1\})$, where $\Pi_{\mathrm{r}}^{(0)}:=\emptyset$. Finally, the cardinality of index set $\Pi_{\mathrm{r}}^{(\kappa)}$ is denoted as $\left|\Pi_{\mathrm{r}}^{(\kappa)}\right|=: M_{\kappa}$.

Let $\operatorname{Pr}\left\{\mathcal{E}^{(\alpha)} \mid P_{\mathrm{S}}, \sigma_{\mathrm{i}, \mathrm{D}}^{2}\right\}$ and $\operatorname{Pr}\left\{\mathcal{E}^{\left(\beta_{\kappa}\right)} \mid P_{\mathrm{S}}\right\}$ denote the conditional probabilities associated with event $\mathcal{E}^{(\alpha)}$ and $\mathcal{E}^{\left(\beta_{\kappa}\right)}$, re- 
spectively, given a fixed source transmit power $P_{\mathrm{S}}$ and a fixed interference power $\sigma_{\mathrm{i}, \mathrm{D}}^{2}$ :

$$
\begin{aligned}
\operatorname{Pr}\left\{\mathcal{E}^{(\alpha)} \mid P_{\mathrm{S}}, \sigma_{\mathrm{i}, \mathrm{D}}^{2}\right\}:=\operatorname{Pr}\left\{\gamma_{\mathrm{S} \rightarrow \mathrm{D}} \geq \gamma_{\mathrm{D}, \text { target }} \mid P_{\mathrm{S}}, \sigma_{\mathrm{i}, \mathrm{D}}^{2}\right\} \\
\operatorname{Pr}\left\{\mathcal{E}^{\left(\beta_{\kappa}\right)} \mid P_{\mathrm{S}}\right\}:=\prod_{i \in \Pi_{\mathrm{r}}^{(\kappa)}} \operatorname{Pr}\left\{\prod_{\left.\mathrm{S} \rightarrow \mathrm{R}_{i} \geq \gamma_{\mathrm{th}} \mid P_{\mathrm{S}}\right\}} \operatorname{Pr}\left\{\gamma_{\mathrm{S} \rightarrow \mathrm{R}_{i}}<\gamma_{\mathrm{th}} \mid P_{\mathrm{S}}\right\} .\right.
\end{aligned}
$$

Moreover, let us denote the conditional CDFs of $\gamma_{\mathrm{D}}$ associated with event $\mathcal{E}^{(\alpha)}$ and event $\mathcal{E}^{\left(\beta_{\kappa}\right)}$ as $C^{(\alpha)}\left(\gamma_{\mathrm{D}} \mid P_{\mathrm{S}}, \sigma_{\mathrm{i}, \mathrm{D}}^{2}\right)$ and $C^{\left(\beta_{\kappa}\right)}\left(\gamma_{\mathrm{D}} \mid P_{\mathrm{S}}, \sigma_{\mathrm{i}, \mathrm{D}}^{2}, P_{\mathrm{R}_{i_{1}}}, \ldots, P_{\mathrm{R}_{i_{M_{\kappa}}}}\right)$, respectively, and let $p_{1}\left(P_{\mathrm{S}}\right), p_{2}\left(\sigma_{\mathrm{i}, \mathrm{D}}^{2}\right)$, and $p_{3, i}\left(P_{\mathrm{R}_{i}}\right)$ denote the probability density functions (PDFs) of $P_{\mathrm{S}}, \sigma_{\mathrm{i}, \mathrm{D}}^{2}$, and $P_{\mathrm{R}_{i}}$, respectively. ${ }^{5}$ With these definitions, the average CDF of the overall MRC output SINR $\gamma_{\mathrm{D}}$ at the destination node can be expressed as:

$$
\begin{aligned}
& \bar{C}\left(\gamma_{\mathrm{D}}\right)=\int_{0}^{\infty} \int_{0}^{P_{\mathrm{S}, \max }} \operatorname{Pr}\left\{\mathcal{E}^{(\alpha)} \mid P_{\mathrm{S}}, \sigma_{\mathrm{i}, \mathrm{D}}^{2}\right\} \\
& \times C^{(\alpha)}\left(\gamma_{\mathrm{D}} \mid P_{\mathrm{S}}, \sigma_{\mathrm{i}, \mathrm{D}}^{2}\right) \cdot p_{1}\left(P_{\mathrm{S}}\right) p_{2}\left(\sigma_{\mathrm{i}, \mathrm{D}}^{2}\right) \mathrm{d} P_{\mathrm{S}} \mathrm{d} \sigma_{\mathrm{i}, \mathrm{D}}^{2} \\
& +\int_{0}^{\infty} \int_{0}^{P_{\mathrm{S}, \max }}\left(1-\operatorname{Pr}\left\{\mathcal{E}^{(\alpha)} \mid P_{\mathrm{S}}, \sigma_{\mathrm{i}, \mathrm{D}}^{2}\right\}\right)
\end{aligned}
$$

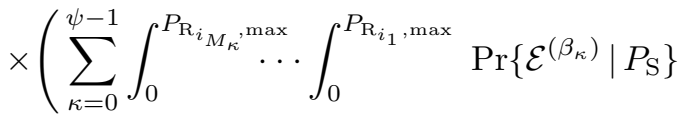

$$
\begin{aligned}
& \times C^{\left(\beta_{\kappa}\right)}\left(\gamma_{\mathrm{D}} \mid P_{\mathrm{S}}, \sigma_{\mathrm{i}, \mathrm{D}}^{2}, P_{\mathrm{R}_{i_{1}}}, \ldots, P_{\mathrm{R}_{i_{M_{\kappa}}}}\right) \\
& \left.\times p_{3, i_{1}}\left(P_{\mathrm{R}_{i_{1}}}\right) \cdots p_{3, i_{M_{\kappa}}}\left(P_{\mathrm{R}_{i_{M_{\kappa}}}}\right) \mathrm{d} P_{\mathrm{R}_{i_{1}}} \cdots \mathrm{d} P_{\mathrm{R}_{i_{M_{\kappa}}}}\right) \\
& \times p_{1}\left(P_{\mathrm{S}}\right) p_{2}\left(\sigma_{\mathrm{i}, \mathrm{D}}^{2}\right) \mathrm{d} P_{\mathrm{S}} \mathrm{d} \sigma_{\mathrm{i}, \mathrm{D}}^{2} .
\end{aligned}
$$

西

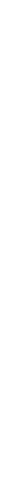

In the following, we provide closed-form expressions for the conditional event probabilities (7) and (8), the conditional $\operatorname{CDFs} C^{(\alpha)}\left(\gamma_{\mathrm{D}} \mid \cdot\right)$ and $C^{\left(\beta_{\kappa}\right)}\left(\gamma_{\mathrm{D}} \mid \cdot\right)$, as well as the PDFs $p_{1}(\cdot), p_{2}(\cdot), p_{3, i}(\cdot)$. Special notations introduced in the sequel are summarized in Table I.

\section{A. Conditional Event Probabilities (7) and (8)}

The conditional event probability (7) can be expressed as

$$
\begin{aligned}
& \operatorname{Pr}\left\{\mathcal{E}^{(\alpha)} \mid P_{\mathrm{S}}, \sigma_{\mathrm{i}, \mathrm{D}}^{2}\right\} \\
& \quad 1-\operatorname{Pr}\left\{\alpha_{\mathrm{S}, \mathrm{D}}<\frac{\gamma_{\mathrm{D}, \text { target }}\left(\sigma_{\mathrm{i}, \mathrm{D}}^{2}+\sigma_{\mathrm{n}, \mathrm{D}}^{2}\right)}{P_{\mathrm{S}}} \mid P_{\mathrm{S}}, \sigma_{\mathrm{i}, \mathrm{D}}^{2}\right\} .
\end{aligned}
$$

According to the Rayleigh-fading assumption, the PDF of $\alpha_{\mathrm{S}, \mathrm{D}}$ is given by [9, Ch. 14.5]

$$
p_{\alpha_{\mathrm{S}, \mathrm{D}}}\left(\alpha_{\mathrm{S}, \mathrm{D}}\right)=\sum_{l=0}^{L_{\mathrm{S}, \mathrm{D}}} \frac{w_{l, \mathrm{~S}, \mathrm{D}}}{\sigma_{l, \mathrm{~S}, \mathrm{D}}^{2}} \exp \left(-\frac{\alpha_{\mathrm{S}, \mathrm{D}}}{\sigma_{l, \mathrm{~S}, \mathrm{D}}^{2}}\right) .
$$

Integration of (11) and evaluation of the resulting $\mathrm{CDF}$ at $\alpha_{\mathrm{S}, \mathrm{D}}=\gamma_{\mathrm{D}, \text { target }}\left(\sigma_{\mathrm{i}, \mathrm{D}}^{2}+\sigma_{\mathrm{n}, \mathrm{D}}^{2}\right) / P_{\mathrm{S}}$ yields

$$
\begin{aligned}
& \operatorname{Pr}\left\{\mathcal{E}^{(\alpha)} \mid P_{\mathrm{S}}, \sigma_{\mathrm{i}, \mathrm{D}}^{2}\right\}= \\
& \quad 1-\sum_{l=0}^{L_{\mathrm{S}, \mathrm{D}}} w_{l, \mathrm{~S}, \mathrm{D}}\left(1-\exp \left(-\frac{\gamma_{\mathrm{D}, \text { target }}}{\bar{\gamma}_{l, \mathrm{~S} \rightarrow \mathrm{D}}}\right)\right) .
\end{aligned}
$$

\footnotetext{
${ }^{5}$ Note that $P_{\mathrm{S}}, \sigma_{\mathrm{i}, \mathrm{D}}^{2}$, and $P_{\mathrm{R}_{i}}$ are functions of the channel energies $\alpha_{\mathrm{S}, \mathrm{U}_{\mathrm{rx}}}$, $\alpha_{\mathrm{U}_{\mathrm{tx}}, \mathrm{D}}$, and $\alpha_{\mathrm{R}_{i}, \mathrm{U}_{\mathrm{rx}}}$, respectively. Moreover, in the case of the FD-TPA scheme the relay transmit powers $P_{\mathrm{R}_{i}}$ are statistically independent.
}

The conditional event probability (8) can be expressed as

$$
\begin{aligned}
\operatorname{Pr}\left\{\mathcal{E}^{\left(\beta_{\kappa}\right)} \mid P_{\mathrm{S}}\right\}= & \prod_{i \in \Pi_{\mathrm{r}}^{(\kappa)}}\left(1-\operatorname{Pr}\left\{\mu_{\mathrm{S}, \mathrm{R}_{i}}<\frac{\gamma_{\text {th }}}{P_{\mathrm{S}}} \mid P_{\mathrm{S}}\right\}\right) \\
& \prod_{i \notin \Pi_{\mathrm{r}}^{(\kappa)}} \operatorname{Pr}\left\{\mu_{\mathrm{S}, \mathrm{R}_{i}}<\frac{\gamma_{\mathrm{th}}}{P_{\mathrm{S}}} \mid P_{\mathrm{S}}\right\}
\end{aligned}
$$

where $\mu_{\mathrm{S}, \mathrm{R}_{i}}:=\alpha_{\mathrm{S}, \mathrm{R}_{i}} /\left(\sigma_{\mathrm{i}, \mathrm{R}_{i}}^{2}+\sigma_{\mathrm{n}, \mathrm{R}_{i}}^{2}\right)$. The PDF of $\alpha_{\mathrm{S}, \mathrm{R}_{i}}$ is of the same form as (11). Moreover, $\sigma_{\mathrm{i}, \mathrm{R}_{i}}^{2}=P_{\mathrm{U}_{\mathrm{tx}}} \alpha_{\mathrm{U}_{\mathrm{tx}}, \mathrm{R}_{i}} / N_{\mathrm{sp}}$ with $p_{\alpha_{\mathrm{U}_{\mathrm{tx}}, \mathrm{R}_{i}}}\left(\alpha_{\mathrm{U}_{\mathrm{tx}}, \mathrm{R}_{i}}\right)=\exp \left(-\alpha_{\mathrm{U}_{\mathrm{tx}}, \mathrm{R}_{i}} / \sigma_{0, \mathrm{U}_{\mathrm{tx}}, \mathrm{R}_{i}}^{2}\right) / \sigma_{0, \mathrm{U}_{\mathrm{tx}}, \mathrm{R}_{i}}^{2}{ }^{6}$ Based on this, the PDF of $\mu_{\mathrm{S}, \mathrm{R}_{i}}$ is given by

$$
\begin{aligned}
& p_{\mu_{\mathrm{S}, \mathrm{R}_{i}}}\left(\mu_{\mathrm{S}, \mathrm{R}_{i}}\right)=C_{1, \mathrm{R}_{i}} \sum_{l=0}^{L_{\mathrm{S}, \mathrm{R}_{i}}} \frac{w_{l, \mathrm{~S}, \mathrm{R}_{i}}}{\sigma_{l, \mathrm{~S}, \mathrm{R}_{i}}^{2}} \\
& \times \frac{\left(C_{1, \mathrm{R}_{i}}+\mu_{\mathrm{S}, \mathrm{R}_{i}} / \sigma_{l, \mathrm{~S}, \mathrm{R}_{i}}^{2}\right) \sigma_{\mathrm{n}, \mathrm{R}_{i}}^{2}+1}{\left(C_{1, \mathrm{R}_{i}}+\mu_{\mathrm{S}, \mathrm{R}_{i}} / \sigma_{l, \mathrm{~S}, \mathrm{R}_{i}}^{2}\right)^{2}} \exp \left(-\frac{\sigma_{\mathrm{n}, \mathrm{R}_{i}}^{2} \mu_{\mathrm{S}, \mathrm{R}_{i}}}{\sigma_{l, \mathrm{~S}, \mathrm{R}_{i}}^{2}}\right) .
\end{aligned}
$$

Integration of (14) and evaluation of the resulting $\mathrm{CDF}$ at $\mu_{\mathrm{S}, \mathrm{R}_{i}}=\gamma_{\mathrm{th}} / P_{\mathrm{S}}$ yields

$$
\operatorname{Pr}\left\{\mu_{\mathrm{S}, \mathrm{R}_{i}}<\frac{\gamma_{\mathrm{th}}}{P_{\mathrm{S}}} \mid P_{\mathrm{S}}\right\}=\sum_{l=0}^{L_{\mathrm{S}, \mathrm{R}_{i}}} w_{l, \mathrm{~S}, \mathrm{R}_{i}} \cdot\left(1-K_{l, \mathrm{R}_{i}}\right) .
$$

Combining (15) with (13) yields the desired closed-form expression for $\operatorname{Pr}\left\{\mathcal{E}^{\left(\beta_{\kappa}\right)} \mid P_{\mathrm{S}}\right\}$.

\section{B. Conditional CDFs $C^{(\alpha)}\left(\gamma_{\mathrm{D}} \mid \cdot\right)$ and $C^{\left(\beta_{\kappa}\right)}\left(\gamma_{\mathrm{D}} \mid \cdot\right)$}

Consider first the case of non-cooperative transmission, where no relays are available. In this case, the PDF of $\gamma_{\mathrm{D}}=\gamma_{\mathrm{S} \rightarrow \mathrm{D}}$, given a fixed source transmit power $P_{\mathrm{S}}$ and a fixed interference power $\sigma_{\mathrm{i}, \mathrm{D}}^{2}$ at the destination node, is of the same form as (11). The corresponding CDF can be calculated as

$$
C_{\mathrm{nc}}\left(\gamma_{\mathrm{D}} \mid P_{\mathrm{S}}, \sigma_{\mathrm{i}, \mathrm{D}}^{2}\right)=\sum_{l=0}^{L_{\mathrm{S}, \mathrm{D}}} w_{l, \mathrm{~S}, \mathrm{D}}\left(1-\exp \left(-\frac{\gamma_{\mathrm{D}}}{\bar{\gamma}_{l, \mathrm{~S} \rightarrow \mathrm{D}}}\right)\right) \text {. }
$$

Next, consider relaying case $(\alpha)$, where the source node is able to accomplish the desired target SINR $\gamma_{\mathrm{D} \text {,target }}$ on its own. By definition we have $\gamma_{D}=\gamma_{S \rightarrow D} \geq \gamma_{D}$,target. Based on (16), we therefore obtain the following expression for the conditional $\operatorname{CDF} C^{(\alpha)}\left(\gamma_{\mathrm{D}} \mid P_{\mathrm{S}}, \sigma_{\mathrm{i}, \mathrm{D}}^{2}\right)$ :

$$
\begin{aligned}
& C^{(\alpha)}\left(\gamma_{\mathrm{D}} \mid P_{\mathrm{S}}, \sigma_{\mathrm{i}, \mathrm{D}}^{2}\right)= \\
& \left\{\begin{array}{cc}
0 & \gamma_{\mathrm{D}}<\gamma_{\mathrm{D}, \text { target }} \\
1+K_{\alpha} \cdot\left(C_{\mathrm{nc}}\left(\gamma_{\mathrm{D}} \mid P_{\mathrm{S}}, \sigma_{\mathrm{i}, \mathrm{D}}^{2}\right)-\varphi_{\mathrm{S}, \mathrm{D}}\right), & \gamma_{\mathrm{D}} \geq \gamma_{\mathrm{D}, \text { target }}
\end{array}\right.
\end{aligned}
$$

Note that for $\gamma_{\mathrm{D}}<\gamma_{\mathrm{D} \text {, target }}$ the first integral in (9) becomes zero, as $C^{(\alpha)}\left(\gamma_{\mathrm{D}} \mid P_{\mathrm{S}}, \sigma_{\mathrm{i}, \mathrm{D}}^{2}\right)$ is zero for $\gamma_{\mathrm{D}}<\gamma_{\mathrm{D} \text {,target }}$. Next, we consider relaying case $(\beta)$, where the source node is not able to accomplish the desired target SINR $\gamma_{\mathrm{D} \text {, target }}$ on its own. For the special case $\left(\beta_{0}\right)$, where no relay is able to decode the message from the source node correctly $\left(\Pi_{\mathrm{r}}=\Pi_{\mathrm{r}}^{(0)}=\emptyset\right)$, we have $\gamma_{\mathrm{D}}=\gamma_{\mathrm{S} \rightarrow \mathrm{D}}<\gamma_{\mathrm{D} \text {,target }}$. We thus obtain the following expression for the conditional $\mathrm{CDF} C^{\left(\beta_{0}\right)}\left(\gamma_{\mathrm{D}} \mid P_{\mathrm{S}}, \sigma_{\mathrm{i}, \mathrm{D}}^{2}\right)$ :

$$
\begin{gathered}
C^{\left(\beta_{0}\right)}\left(\gamma_{\mathrm{D}} \mid P_{\mathrm{S}}, \sigma_{\mathrm{i}, \mathrm{D}}^{2}\right)= \\
\left\{\begin{array}{cl}
K_{\beta} C_{\mathrm{nc}}\left(\gamma_{\mathrm{D}} \mid P_{\mathrm{S}}, \sigma_{\mathrm{i}, \mathrm{D}}^{2}\right), & \gamma_{\mathrm{D}}<\gamma_{\mathrm{D}, \text { target }} \\
1 & \gamma_{\mathrm{D}} \geq \gamma_{\mathrm{D}, \text { target }}
\end{array}\right.
\end{gathered}
$$

\footnotetext{
${ }^{6}$ Recall that all links associated with the primary users are modeled by frequency-flat fading $\left(L_{\mathrm{X}, \mathrm{Y}}=0\right)$, as the bandwidth ratio $\rho=B_{\mathrm{U}} / B_{\mathrm{CR}}$ was assumed to be small.
} 
If a certain non-zero subset $\Pi_{\mathrm{r}}^{(\kappa)} \subseteq\left\{1, \ldots, N_{\mathrm{r}}\right\}$ of relays is able to decode the message from the source node correctly, $\gamma_{\mathrm{D}}$ is given by $\gamma_{\mathrm{D}}=\gamma_{\mathrm{S} \rightarrow \mathrm{D}}+\gamma_{\mathrm{R} \rightarrow \mathrm{D}}$ while $\gamma_{\mathrm{S} \rightarrow \mathrm{D}}<\gamma_{\mathrm{D} \text {, target }}$. Therefore, for a fixed source transmit power $P_{\mathrm{S}}$, a fixed interference power $\sigma_{\mathrm{i}, \mathrm{D}}^{2}$ at the destination node, and fixed relay transmit powers $P_{\mathrm{R}_{i}}\left(i \in \mathbb{I}_{\mathrm{r}}^{(\kappa)}\right)$, the PDF of $\gamma_{\mathrm{D}}$ can be calculated by convolving the constrained PDF of $\gamma_{\mathrm{S} \rightarrow \mathrm{D}}$ with the PDF of $\gamma_{\mathrm{R} \rightarrow \mathrm{D}}$ :

$$
p_{\gamma_{\mathrm{R} \rightarrow \mathrm{D}}}\left(\gamma_{\mathrm{R} \rightarrow \mathrm{D}}\right)=\sum_{i \in \Pi_{\mathrm{r}}^{(\kappa)}} \sum_{l=0}^{L_{\mathrm{R}, \mathrm{D}}} \frac{\tilde{w}_{l, \mathrm{R}_{i}, \mathrm{D}}}{\bar{\gamma}_{l, \mathrm{R}_{i} \rightarrow \mathrm{D}}} \exp \left(-\frac{\gamma_{\mathrm{R} \rightarrow \mathrm{D}}}{\bar{\gamma}_{l, \mathrm{R}_{i} \rightarrow \mathrm{D}}}\right) \text {. }
$$

For simplicity, we have assumed in (19) that $L_{\mathrm{R}_{i}, \mathrm{D}}=: L_{\mathrm{R}, \mathrm{D}}$ for all $i \in \mathbb{I}_{\mathrm{r}}^{(\kappa)}$. Integration of the resulting PDF finally yields the conditional CDF $C^{\left(\beta_{\kappa}\right)}\left(\gamma_{\mathrm{D}} \mid P_{\mathrm{S}}, \sigma_{\mathrm{i}, \mathrm{D}}^{2}, P_{\mathrm{R}_{i_{1}}}, \ldots, P_{\mathrm{R}_{i_{M_{\kappa}}}}\right)$ :

$$
\begin{aligned}
& C^{\left(\beta_{\kappa}\right)}\left(\gamma_{\mathrm{D}} \mid P_{\mathrm{S}}, \sigma_{\mathrm{i}, \mathrm{D}}^{2}, P_{\mathrm{R}_{i_{1}}}, \ldots, P_{\mathrm{R}_{i_{M_{\kappa}}}}\right)= \\
& \left\{\begin{array}{l}
K_{\beta} \sum_{i \in \Pi_{\mathrm{r}}^{(\kappa)}} \sum_{l=0}^{L_{\mathrm{S}, \mathrm{D}}} \sum_{\lambda=0}^{L_{\mathrm{R}, \mathrm{D}}} v_{l, \lambda, i}\left[\bar{\gamma}_{\lambda, \mathrm{R}_{i} \rightarrow \mathrm{D}}\left(\exp \left(-\frac{\gamma_{\mathrm{D}}}{\bar{\gamma}_{\lambda, \mathrm{R}_{i} \rightarrow \mathrm{D}}}\right)-1\right)\right. \\
\quad-\bar{\gamma}_{l, \mathrm{~S} \rightarrow \mathrm{D}}\left(\exp \left(-\frac{\gamma_{\mathrm{D}}}{\bar{\gamma}_{l, \mathrm{~S} \rightarrow \mathrm{D}}}\right)-1\right), \quad \gamma_{\mathrm{D}}<\gamma_{\mathrm{D}, \text { target }} \\
K_{\beta} \sum_{i \in \Pi_{\mathrm{r}}^{(\kappa)}} \sum_{l=0}^{L_{\mathrm{S}, \mathrm{D}}} \sum_{\lambda=0}^{L_{\mathrm{R}, \mathrm{D}}} v_{l, \lambda, i}\left[\left(\bar{\gamma}_{\lambda, \mathrm{R}_{i} \rightarrow \mathrm{D}}-\bar{\gamma}_{l, \mathrm{~S} \rightarrow \mathrm{D}}\right)\right. \\
\quad \times\left(\exp \left(-\frac{\gamma_{\mathrm{D}, \text { target }}}{\bar{\gamma}_{l, \mathrm{~S} \rightarrow \mathrm{D}}}\right)-1\right)-\bar{\gamma}_{\lambda, \mathrm{R}_{i} \rightarrow \mathrm{D}} \\
\left.\quad \times\left(\exp \left(C_{3, l, \lambda, \mathrm{R}_{i}} \gamma_{\mathrm{D}, \text { target }}\right)-1\right) \exp \left(-\frac{\gamma_{\mathrm{D}}}{\bar{\gamma}_{\lambda, \mathrm{R}_{i} \rightarrow \mathrm{D}}}\right)\right], \\
\gamma_{\mathrm{D}} \geq \gamma_{\mathrm{D}, \text { target }} .
\end{array}\right.
\end{aligned}
$$

C. PDFs $p_{1}(\cdot), p_{2}(\cdot), p_{3, i}(\cdot)$ in (9)

The PDF $p_{1}\left(P_{\mathrm{S}}\right)$ of the source transmit power $P_{\mathrm{S}}$ according to (1) can be evaluated as

$$
\begin{aligned}
& p_{1}\left(P_{\mathrm{S}}\right)= \\
& \left\{\begin{array}{cl}
\frac{C_{4, \mathrm{~S}}}{P_{\mathrm{S}}^{2}} \exp \left(-\frac{C_{4, \mathrm{~S}}}{P_{\mathrm{S}}}\right)+\left(1-\exp \left(-\frac{C_{4, \mathrm{~S}}}{P_{\mathrm{S}, \max }}\right)\right) & P_{\mathrm{S}, \max } \\
\times \delta_{0}\left(P_{\mathrm{S}}-P_{\mathrm{S}, \max }\right), & P_{\mathrm{S}} \leq P_{\mathrm{S}}>P_{\mathrm{S}, \max } .
\end{array}\right.
\end{aligned}
$$

Here $\delta_{0}\left(P_{\mathrm{S}}-P_{\mathrm{S}, \max }\right)$ denotes a Dirac impulse at $P_{\mathrm{S}}=P_{\mathrm{S}, \max }$. In order to arrive at (21), we have used that the PDF of $\alpha_{\mathrm{S}, \mathrm{U}_{\mathrm{rx}}}$ is given by $p_{\alpha_{\mathrm{S}, \mathrm{U}_{\mathrm{rx}}}}\left(\alpha_{\mathrm{S}, \mathrm{U}_{\mathrm{rx}}}\right)=\exp \left(-\alpha_{\mathrm{S}, \mathrm{U}_{\mathrm{rx}}} / \sigma_{0, \mathrm{~S}, \mathrm{U}_{\mathrm{rx}}}^{2}\right) / \sigma_{0, \mathrm{~S}, \mathrm{U}_{\mathrm{rx}}}^{2}$. If the interference constraint vanishes (i.e., $\rho \sigma_{0, \mathrm{~S}, \mathrm{U}_{\mathrm{rx}}}^{2} \rightarrow 0$ ), we obtain $p_{1}\left(P_{\mathrm{S}}\right)=\delta_{0}\left(P_{\mathrm{S}}-P_{\mathrm{S}, \max }\right)$, as expected.

The PDF $p_{2}\left(\sigma_{\mathrm{i}, \mathrm{D}}^{2}\right)$ of the interference power $\sigma_{\mathrm{i}, \mathrm{D}}^{2}$ at the destination node is given by

$$
p_{2}\left(\sigma_{\mathrm{i}, \mathrm{D}}^{2}\right)=C_{1, \mathrm{D}} \mathrm{e}^{-C_{1, \mathrm{D}} \sigma_{\mathrm{i}, \mathrm{D}}^{2}} .
$$

To arrive at (22), we have used that $\sigma_{\mathrm{i}, \mathrm{D}}^{2}=P_{\mathrm{U}_{\mathrm{tx}}} \alpha_{\mathrm{U}_{\mathrm{tx}}, \mathrm{D}} / N_{\mathrm{sp}}$, while the PDF of $\alpha_{\mathrm{U}_{\mathrm{tx}}, \mathrm{D}}$ is given by $p_{\alpha_{\mathrm{U}_{\mathrm{tx}}, \mathrm{D}}}\left(\alpha_{\mathrm{U}_{\mathrm{tx}}, \mathrm{D}}\right)=$ $\exp \left(-\alpha_{\mathrm{U}_{\mathrm{tx}}, \mathrm{D}} / \sigma_{0, \mathrm{U}_{\mathrm{tx}}, \mathrm{D}}^{2}\right) / \sigma_{0, \mathrm{U}_{\mathrm{tx}}, \mathrm{D}}^{2}$.

Finally, similar to (21) one obtains the following PDF for the relay transmit power $P_{\mathrm{R}_{i}}\left(i \in \mathbb{I}_{\mathrm{r}}^{(\kappa)}\right)$ :

$$
\left\{\begin{array}{cc}
\frac{C_{4, \mathrm{R}_{i}}}{N_{\mathrm{r}}^{\prime} P_{\mathrm{R}_{i}}^{2}} \exp \left(-\frac{C_{4, \mathrm{R}_{i}}}{N_{\mathrm{r}}^{\prime} P_{\mathrm{R}_{i}}}\right)+\left(1-\exp \left(-\frac{C_{4, \mathrm{R}_{i}}}{N_{\mathrm{r}}^{\prime} P_{\mathrm{R}_{i}, \max }}\right)\right) \\
\times \delta_{0}\left(P_{\mathrm{R}_{i}}-P_{\mathrm{R}_{i}, \max }\right), & P_{\mathrm{R}_{i}} \leq P_{\mathrm{R}_{i}, \max } \\
0 & P_{\mathrm{R}_{i}}>P_{\mathrm{R}_{i}, \max } .
\end{array}\right.
$$

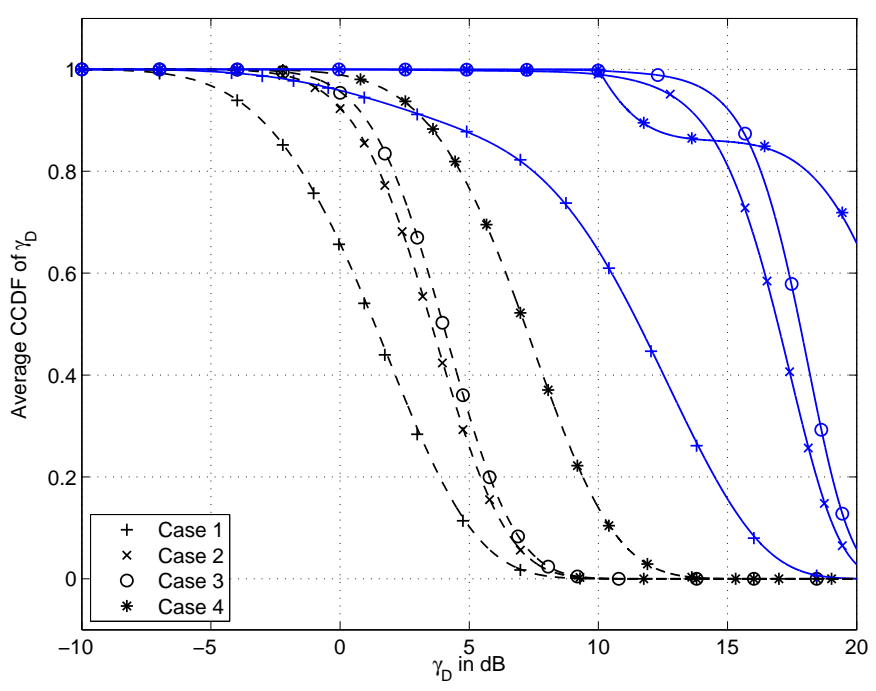

Fig. 2. Average CCDF $1-\bar{C}\left(\gamma_{\mathrm{D}}\right)$ of the overall MRC output SINR $\gamma_{\mathrm{D}}$ at the destination node for different cases. Case 1: $\bar{\gamma}_{0, \mathrm{~S} \rightarrow \mathrm{D}}=5 \mathrm{~dB}, d=3, N_{\mathrm{sp}}=10$; Case 2: $\bar{\gamma}_{0, \mathrm{~S} \rightarrow \mathrm{D}}=5 \mathrm{~dB}, d=12, N_{\mathrm{sp}}=10$; Case $3: \bar{\gamma}_{0, \mathrm{~S} \rightarrow \mathrm{D}}=5 \mathrm{~dB}, d=12$, $N_{\mathrm{sp}}=20$; Case 4: $\bar{\gamma}_{0, \mathrm{~S} \rightarrow \mathrm{D}}=10 \mathrm{~dB}, d=12, N_{\mathrm{sp}}=10$. Lines represent (semi-)analytical results obtained by means of Monte-Carlo integration of (9), whereas markers represent simulation results. Dashed lines: non-cooperative transmission; solid lines: FD-TPA scheme.

Again, $p_{3, i}\left(P_{\mathrm{R}_{i}}\right)=\delta_{0}\left(P_{\mathrm{R}_{i}}-P_{\mathrm{R}_{i}, \max }\right)$ for $\rho \sigma_{0, \mathrm{R}_{i}, \mathrm{U}_{\mathrm{rx}}}^{2} \rightarrow 0$.

Based on the above closed-form expressions for the conditional event probabilities, the corresponding conditional CDFs, and the PDFs $p_{1}\left(P_{\mathrm{S}}\right), p_{2}\left(\sigma_{\mathrm{i}, \mathrm{D}}^{2}\right)$, and $p_{3, i}\left(P_{\mathrm{R}_{i}}\right)$, a further evaluation of the average $\operatorname{CDF} \bar{C}\left(\gamma_{\mathrm{D}}\right)$ of the overall MRC output SINR $\gamma_{\mathrm{D}}$ at the destination node (cf. (9)) appears to be difficult. $^{7}$ In the following section, we will therefore apply a MonteCarlo integration method, in order to evaluate $\bar{C}\left(\gamma_{\mathrm{D}}\right)$. We also note that an extension of the presented analysis to the case of the optimal centralized solution resulting from the linear program (5) does not seem feasible, since there is no closed-form expression for the relay transmit powers $P_{\mathrm{R}_{i}}$.

\section{Numerical Performance Results}

In the following, the performance of the FD-TPA scheme is evaluated and compared to that of non-cooperative transmission and the optimal centralized (OC) TPA solution.

As in the analysis in Section III, quasi-static Rayleigh fading is assumed. All link lengths are normalized with respect to the distance between source and destination, i.e., $d_{\mathrm{ref}}:=d_{\mathrm{S}, \mathrm{D}}$. We choose a path-loss exponent of $p=3$. The average signalto-noise ratio (SNR) of the source-destination link, denoted as $\bar{\gamma}_{0, \mathrm{~S} \rightarrow \mathrm{D}}$, serves as a reference in the sequel. The locations of source and destination are set to $(-0.5,0)$ and $(+0.5,0)$, respectively. The links within the CR network are assumed to have a channel memory length of $L_{\mathrm{X}, \mathrm{Y}}=9$ and an exponentially decaying power profile, according to $\tilde{\sigma}_{l, \mathrm{X}, \mathrm{Y}}^{2} / \tilde{\sigma}_{0, \mathrm{X}, \mathrm{Y}}^{2}=$ $\exp \left(-l / c_{h}\right)$, where we choose $c_{h}:=2$. As an example, we assume that $N_{\mathrm{r}}=5$ relays are available with positions $(0,0)$, $(0, \pm 0.2)$, and $(0, \pm 0.4)$. For simplicity, all nodes within the CR network are assumed to have identical physical properties. To this end, we set $\sigma_{\mathrm{n}, \mathrm{D}}^{2}=\sigma_{\mathrm{n}, \mathrm{R}_{i}}^{2}=: \sigma_{\mathrm{n}}^{2}$ for all in-

\footnotetext{
${ }^{7}$ To this end, the conditional event probabilities and the conditional CDFs in (9) would have to be averaged over the source transmit power $P_{\mathrm{S}}$, the interference power $\sigma_{\mathrm{i}, \mathrm{D}}^{2}$, and the relay transmit powers $P_{\mathrm{R}_{i}}$, utilizing the expressions (21)-(23) for the corresponding PDFs.
} 


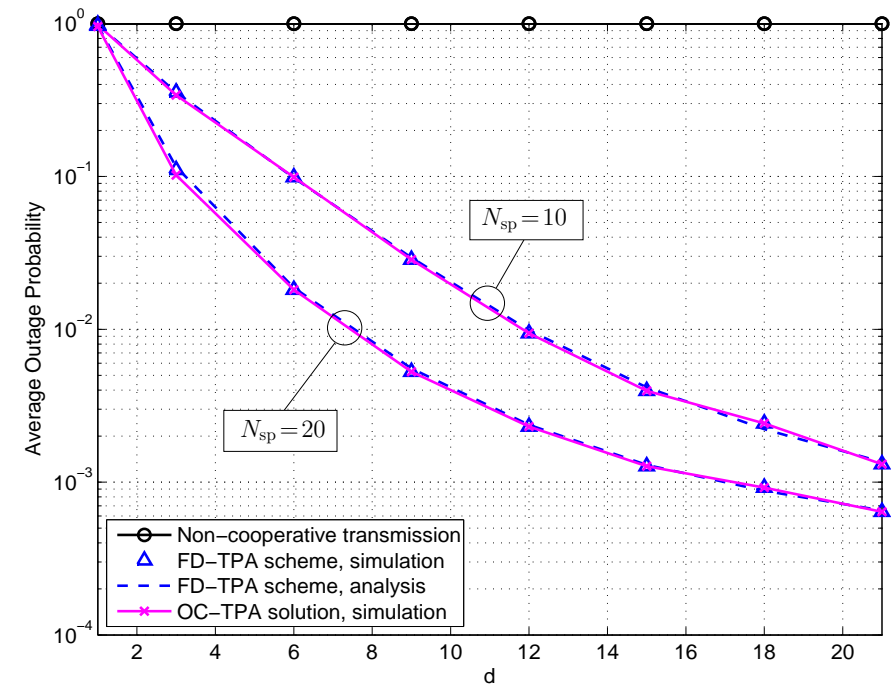

Fig. 3. Average outage probability $\bar{C}\left(\gamma_{\mathrm{D}}\right.$, target $)$ for non-cooperative transmission, the FD-TPA scheme, and the OC-TPA solution as a function of the distance between the primary system and the CR network. For the FD-TPA scheme, dashed lines represent (semi-)analytical results obtained by means of Monte-Carlo integration, whereas markers represent simulation results. The curves for the OC-TPA solution were obtained by means of simulations.

dices $i \in\left\{1, \ldots, N_{\mathrm{r}}\right\}$ and choose (normalized) maximum transmit powers of $P_{\mathrm{S}, \max }=P_{\mathrm{R}_{i}, \text { max }}:=1$ for the source node and the relays. Finally, we set $\gamma_{\mathrm{th}}=10 \mathrm{~dB}$ and $\gamma_{\mathrm{D} \text {, target }}=10 \mathrm{~dB}$.

As discussed earlier, we assume that the average transmit power $P_{\mathrm{U}_{\mathrm{tx}}}$ employed by the primary transmitter is much larger than the maximum transmit powers within the CR network. As an example we set $P_{\mathrm{U}_{\mathrm{tx}}}:=10,000$. Consequently, the primary transmitter needs to be located at some distance from the CR network, in order to allow for secondary spectrum usage. As an example, we consider different positions $(0, d+10)$ and $(0, d)$ of the primary transmitter and receiver, respectively. The maximum sum interference power tolerated by the primary receiver, on the other hand, should be expected to be rather small. As an example, we set $\xi:=0.01$. Finally, the bandwidth ratio between the primary link and the CR network is set to $\rho=0.1$. All simulation results presented in the following have been averaged over $10^{5}$ statistically independent channel realizations.

Fig. 2 displays the average complementary CDF (CCDF) of the overall MRC output SINR $\gamma_{\mathrm{D}}, 1-\bar{C}\left(\gamma_{\mathrm{D}}\right)$, resulting for noncooperative transmission and the FD-TPA scheme, respectively, for four different cases specified in the figure caption. As can be seen, the (semi-)analytical results (dashed/solid lines) based on (9) and (16) are in good accordance with the simulation results (markers). Moreover, it can be seen that for all considered cases the FD-TPA scheme substantially outperforms non-cooperative transmission, as the associated average CCDFs are located significantly further to the right. Furthermore, we note that the performance of both non-cooperative transmission and the FDTPA scheme improves, if (i) the distance $d$ of the primary system from the CR network is increased, (ii) the spreading length $N_{\text {sp }}$ is increased, or (iii) the reference SNR $\bar{\gamma}_{0, \mathrm{~S} \rightarrow \mathrm{D}}$ is increased. However, even for $\bar{\gamma}_{0, \mathrm{~S} \rightarrow \mathrm{D}}=10 \mathrm{~dB}$ and $d=12$ (Case 4), the probability that the target SINR $\gamma_{\mathrm{D} \text {, target }}=10 \mathrm{~dB}$ is accomplished by means of non-cooperative transmission is only about $14 \%$. In the case of the FD-TPA scheme, the plateau within $1-\bar{C}\left(\gamma_{\mathrm{D}}\right)$ for $\bar{\gamma}_{0, \mathrm{~S} \rightarrow \mathrm{D}}=10 \mathrm{~dB}$ (Case 4 ) is due to the relaying process, since in those cases where the source node is not able to accomplish the target SINR $\gamma_{\mathrm{D} \text {, target }}$ on its own, the participating relays maximize $\gamma_{D}$ according to a best-effort strategy. As a consequence, the FD-TPA scheme exhibits a better CCDF curve for $\bar{\gamma}_{0, \mathrm{~S} \rightarrow \mathrm{D}}=5 \mathrm{~dB}$ (Case 2) than for $\bar{\gamma}_{0, \mathrm{~S} \rightarrow \mathrm{D}}=10 \mathrm{~dB}$ (Case 4), as long as $\gamma_{\mathrm{D}} \leq 14 \mathrm{~dB}$.

The advantage of the FD-TPA scheme over non-cooperative transmission is even more apparent in terms of the average outage probability $\mathrm{E}\left\{\operatorname{Pr}\left\{\gamma_{\mathrm{D}}<\gamma_{\mathrm{D} \text {, target }}\right\}\right\}=\bar{C}\left(\gamma_{\mathrm{D} \text {, target }}\right)$. Fig. 3, shows $\bar{C}\left(\gamma_{\mathrm{D} \text {,target }}\right)$ as a function of the distance $d$ between the primary system and the CR network $\left(\bar{\gamma}_{0, S \rightarrow D}=5 \mathrm{~dB}\right.$, $\left.N_{\text {sp }}=10,20\right)$. As can be seen, the FD-TPA scheme substantially outperforms non-cooperative transmission. In particular, while the average outage probability for non-cooperative transmission remains close to one for the entire range of $d$ under consideration (both for $N_{\mathrm{sp}}=10$ and $N_{\mathrm{sp}}=20$ ), the average outage probability for the FD-TPA scheme decreases significantly with growing distance $d$. As earlier, the (semi-)analytical results based on (9) are in good accordance with the simulation results. Finally, simulation results obtained for the OC-TPA solution (resulting from (5)) illustrate that the average outage probability of the FD-TPA scheme is, in fact, very close to the optimum one (throughout the entire range of $d$ ).

\section{Conclusions}

In this paper, a fully decentralized (FD) transmit power allocation (TPA) scheme for relay-assisted cognitive-radio (CR) systems in the presence of an active primary narrowband link has been considered and its performance has been analyzed. The FD-TPA scheme maximizes the signal-to-noiseplus-interference ratio at the destination node of the CR network according to a best-effort strategy, while limiting the interference experienced by the primary receiver. Numerical performance results have shown that the FD-TPA scheme accomplishes substantial performance improvements over noncooperative transmission. Moreover, its performance has been shown to be close to the optimal centralized TPA solution.

For future work, decentralized multi-hop solutions will be interesting, so as to further improve performance (especially when the primary system is close to the CR network).

\section{REFERENCES}

[1] S. Haykin, "Cognitive radio: Brain-empowered wireless communications," IEEE J. Select. Areas Commun., vol. 23, no. 2, pp. 201-220, Feb. 2005.

[2] A. Ghasemi and E. S. Sousa, "Spectrum sensing in cognitive radio networks: Requirements, challenges and design trade-offs," IEEE Commun. Mag., vol. 46, no. 4, pp. 32-39, Apr. 2008.

[3] H. Zhang, X. Zhou, K. Y. Yazdandoost, and I. Chlamtac, "Multiple signal waveforms adaptation in cognitive ultra-wideband radio evolution," IEEE J. Select. Areas Commun., vol. 24, no. 4, pp. 878-884, Apr. 2006.

[4] K. Lee and A. Yener, "Outage performance of cognitive wireless relay networks," in Proc. IEEE Global Telecommun. Conf. (Globecom), San Francisco, California, USA, Nov./Dec. 2006.

[5] A. K. Sadek, K. J. R. Liu, and A. Ephremides, "Cognitive multiple access via cooperation: Protocol design and performance analysis," IEEE Trans. Inform. Theory, vol. 53, no. 10, pp. 3677-3696, Oct. 2007.

[6] J. Mietzner, L. Lampe, and R. Schober, "Distributed transmit power allocation for relay-assisted cognitive-radio systems," in Proc. Asilomar Conf. on Signals, Systems, and Computers, Pacific Grove, California, USA, Nov 2007, pp. 792-796.

[7] C. Sun and K. B. Letaief, "User cooperation in heterogeneous cognitive radio networks with interference reduction," in Proc. IEEE Int. Conf. Commun. (ICC), Beijing, China, May 2008, pp. 3193-3197.

[8] A. F. Molisch, Wireless Communications. Chichester: Wiley/IEEE Press, 2005 .

[9] J. G. Proakis, Digital Communications, 4th ed. New York: McGrawHill, 2001.

[10] W. L. Winston and M. Venkataramanan, Introduction to Mathematical Programming - Operations Research: Volume One. 4th ed. Pacific Grove, CA: Thomson Learning - Brooks/Cole, 2003. 